\title{
Creative product design using biomimetics
}

\author{
J. C. L. Neves \& I. C. M. Francke \\ Natura Inovação e Tecnologia de Produtos Ltda, Cajamar, Brazil
}

\begin{abstract}
Biomimetics is based on the use of biological concepts in the development of innovative technologies, products, and processes. Indeed, nature is a vast source of wisdom, with 3.8 billion years of experience in preserving successful biological strategies and discarding failures. A complete understanding of natural constraints, challenges, and corresponding solutions is however required for these strategies to be used to solve human problems, including the development of new products with minimal use of energy and materials. Additionally, the incorporation of Biomimetics into the industrial process has to be conducted as part of a structured process. In partnership with a design company, we built a structured methodology for the application of Biomimetics in the development of packaging solutions for our cosmetics company. We initially identified 12 demands associated with packaging. Of those, containing was identified as a central demand, which prompted us to ask How does Nature contain liquids? A report describing biological solutions to this problem included drawings and pictures of organisms and potential ideas. This approach resulted in 25 new proposals for the development of new packaging strategies and products. Our results show that it is possible to incorporate the science of Biomimetics into the creative process underlying the development of new strategies and products in a cosmetics company, and possibly in other types of companies as well.
\end{abstract}

Keywords: biomimetics, sustainable packaging, fluid retention, natural functions, key concepts, innovation, creative process.

\section{Introduction}

Several structured processes for product development exist nowadays. The identification of the steps inherent to these processes and their related activities turn these into Cartesian processes in which product development follows a preestablished sequence that is delimited by steps and deliveries, which in turn are 
divided into macro phases of process development. Product development goes through an initial conceptual phase, referred as a Conceptual Design and Information or Conceptual Framework and Design Information according to Detanico et al. [1].

Biomimetics (bio meaning life and mimetic meaning an aptitude for mimicry) is the science that aims to understand successful strategies adopted by Nature to better adapt organisms to life, and then mimic and apply such strategies to solve human problems. Nature's solutions may then be applied to the process of product development and may minimize the energy and material required, creating innovative technologies and processes.

Structured processes are crucial for the development and management of new products and technologies. Structured processes also help in monitoring project execution by establishing activities and responsibilities among teams working on a multidisciplinary project. The incorporation of Biomimetics into these developmental processes, in a structured way, may offer a great opportunity for generating differential products.

\section{Challenges and demands}

In order to apply Biomimetics to the creative process of product development, an understanding of the challenges and demands associated with this task is mandatory. These challenges and demands need to be clearly recognized and their realm and limitations well defined. Finally, the goals associated to this task need to be established.

Before seeking a biological solution to a problem, it is necessary to identify the functions associated to a given challenge. Next, a clear understanding of the existing restrictions in which the product will be used and the conditions it might be exposed to is required. Thus, one should ask about the scenario in which this new product will be adopted. By recognizing and addressing these issues, it is possible to define the focus of the research on Nature's strategies.

In a partnership with the Biomimicry Guild [2], Natura has started a pilot project in Biomimetics aiming to evaluate the potential of applying Biomimetics to product development and innovation processes and the company's technologies. Our starting point was a task that involved a multidisciplinary working group in which several people in the company participated in a survey of the demands associated with this challenge and in validating a packagingrelated challenge. The multidisciplinary working group included workers in Packaging Development, Science and Technology, and other departments in the company.

The basic functions associated with packaging are to contain, to protect, and to transport. But are other demands associated with packaging for which seeking inspiration in Nature may result in relevant innovations? The working group identified 12 demands clearly associated with packaging. 


\subsection{Identifying the demands}

Initially, 12 demands were identified that were related to product development.

These were:

1. Total use of the product (until the last drop);

2. Packaging and logistics (storage);

3. Optimization of resources (minimum use of materials);

4. Production, usage, and disposal (closed loop);

5. Refill-based products (refill);

6. Size optimization (compression);

7. Dispenser solutions (pumps and covers);

8. Order picking;

9. Package functionality (ergonomy);

10. Product usage (experience);

11. Multiples and dosages (multipack);

12. Performance (product).

\subsection{Framing the question}

Once the demands were identified and the packaging-associated challenges defined, the next step was to formulate the question to be posed to Nature. During the debates among individuals in the working team, the need to contain materials in various states was raised as a central issue to be considered when formulating the question. However, How does Nature contain...? is a loose question that offers no clear direction to be followed. Thus, we started by considering the containing of liquids, even though other types of formula, such as powder and creams, are also common in the cosmetic industry.

The first part of the task conducted by the Biomimetics Guild was to raise the following question: How does Nature contain liquids?

\subsubsection{Biomimetics Perspective report - "the inspirational catalog"}

Once the question was raised, the Biomimetics Guild started looking for biological solutions to this problem. Following the methodology applied by the Biomimicry Guild a set of answers were gathered in the Biomimicry Perspectives Report. The report describes possible solutions and strategies found in Nature and includes drawings and pictures and the name of the organism associated with each specific solution and/or strategy. Also, potential ideas for application, taking into account the proposed challenge associated with the design and development of the new product, were also included in the report.

The solutions described in this report were divided into predominately physically based solutions, predominately chemically based solutions, and those combining chemical and physical solutions.

With this reports in hand we then looked for solutions that could be applied to product development and design, and that could address the proposed challenge and demands initially identified. 


\section{Understanding the report}

The adoption of Biomimetics for product development and new packaging strategies was a novel innovative process in our company. Thus, we teamed up with the design agency, Tátil Design de Ideias [3] to develop a methodology that would help us understand how to extract the information contained in the report. Throughout the process, new ideas and concepts that could be applied to our demands for innovation were generated.

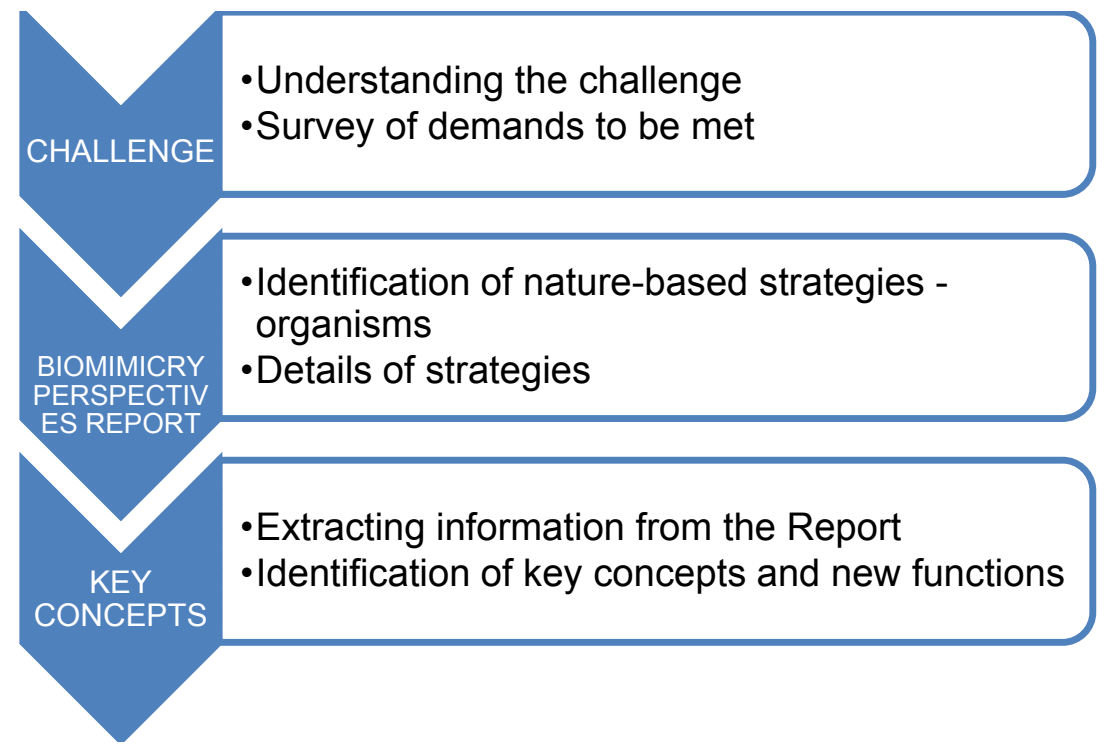

Figure 1: Initial structure of the methodology.

\subsection{Key concepts}

From the 30 nature-based strategies (with each strategy corresponding to a single organism) potentially suitable to address the initial challenge, we analyzed each organism individually and searched for specific words naming key concepts that could characterize each of these organisms. Such words may also characterize the strategies adopted by each organism and may be used to extend our understanding of such strategies, increasing the chances of finding answers for the challenge proposed.

Figure 2 shows how the process starts, from the point where the challenge is identified and onwards. We next built a structured methodology that could help us apply Biomimetics into the innovation process of new packaging solutions. Next, all the possible solutions with creative potential were described in detail and grouped by key concepts. These concepts could also characterize other functions supporting the adaptive strategies identified. We then were able to extend the research by searching for other organisms carrying these newly found 
functions identified through key concepts. In this step of the process, the organisms initially identified were divided into six groups, as illustrated in figure 2 .

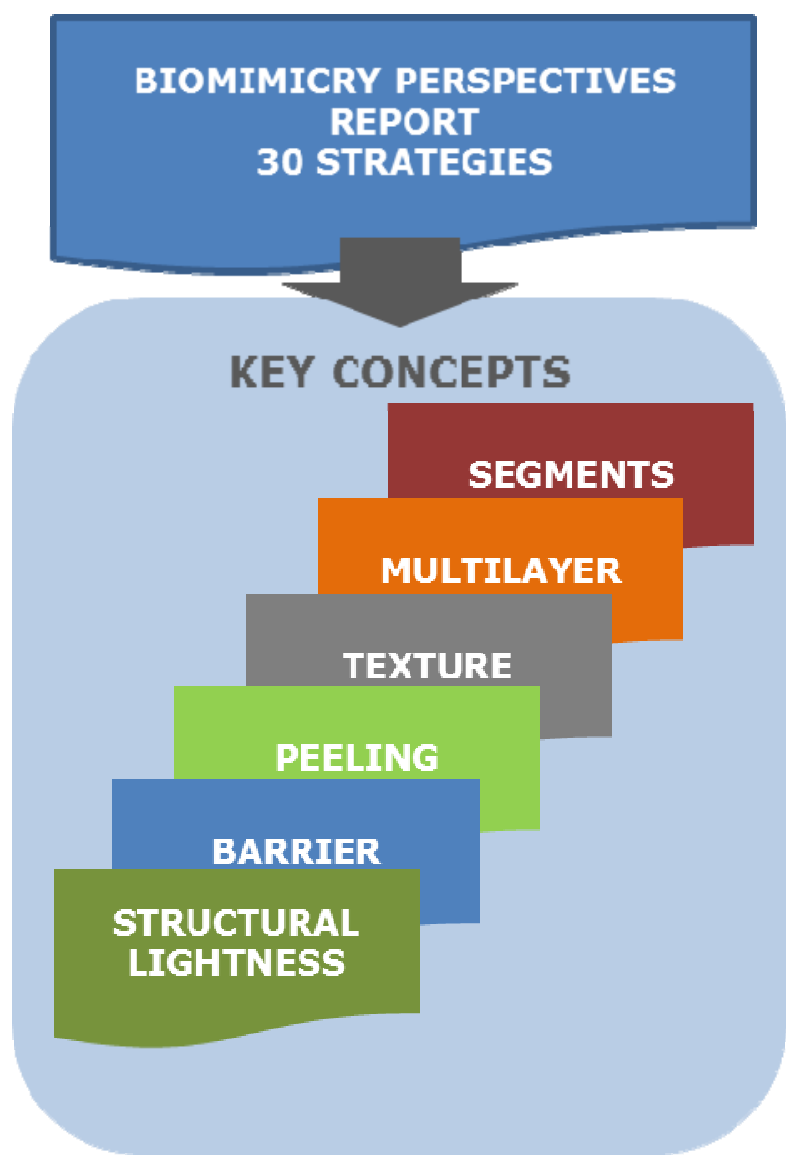

Figure 2: Key concepts identified in the analysis of the initial report.

Of the key concepts identified, we were able to include 11 organisms carrying nature-based strategies that could potentially address the initial challenge. This process amplified the scope initially planned and addressed the challenge associated with a new design or product. This resulted in 41 nature-based strategies adopted as inspiration to address the proposed challenge.

\section{Results}

With all nature-based strategies defined, we proceeded to recognize the needs associated with packaging in order to identify the potential of each strategy in addressing the demands. Figure 3 illustrates the whole process. 


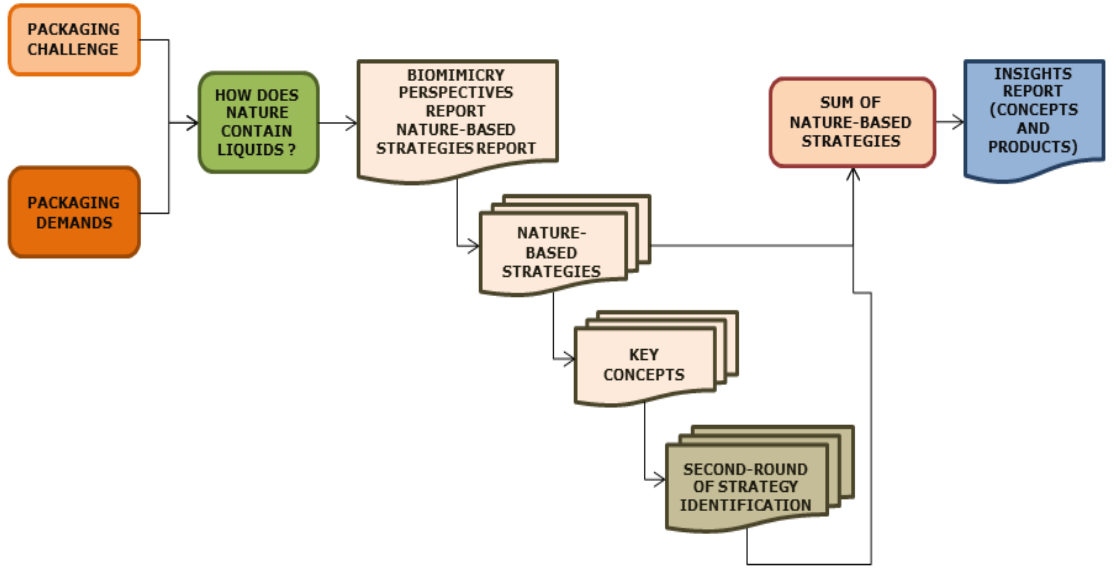

Figure 3: Steps of the structured creative process where Biomimetics is incorporated.

In partnership with Tátil Design de Ideias we undertook the interpretation of the nature-based strategies to understand how each organism works. Next, we developed a creative process in which analogies were drawn between function and potential solutions.

It should be noted that this project also included a group of designers and biologists who interacted with each other for a better understanding of the potential application of nature-based solutions. This project resulted in a second report with 17 proposals for packaging and 8 proposals for new products. The proposals are now under evaluation in the company and may be included in the catalog of projects.

This project also allowed the creation of a research program in Biomimetics in our company as many professionals working in Natura, including designers, biologists, biomedical, pharmaceutical, chemists, physicists, and engineers were trained by the Biomimicry Guild.

\section{Conclusions}

Here we aimed to present the key elements of the rationale adopted in our company for the application of Biomimetics into the process of product development. Our results show that is possible to incorporate the science of Biomimetics into the creative process required for the development of new strategies and products in a cosmetics company, and possibly in other types of companies as well. Our results also show that the structured methodology built in partnership with Tátil Design de Ideias for the application of Biomimetics in our company was very successful as it led to 25 new proposals for the development of new packaging solutions.

We believe that this methodology could be easily adapted and incorporated by other groups working on the R\&D of new products adopt our methodology as 
a means to accelerate the creative process and optimize the development of new products and technologies.

\section{Acknowledgements}

This manuscript was reviewed by a professional science editor and by a native English-speaking copy editor to improve readability (Publicase).

\section{References}

[1] Detanico, F.B., Teixeira, F.G. and Silva, T.K., Biomimetics as a Creative Method for Product Design, Universidade Federal do Rio Grande do Sul, Porto Alegre, Brasil, 2010.

[2] Biomimicry Guild - Natura Biomimicry Perspectives Report, Helena, Montana, USA, 2010.

[3] Tátil Design de Ideias - Tátil Report: Natura and Biomimetic Project, Rio de Janeiro, Brasil, 2010. 